\title{
Nurse Sows' Reproductive Performance in Different Parities and Lifetime Productivity in Spain
}

\author{
Ryosuke Iida $^{1}$, Yu Yatabe ${ }^{1}$, Carlos Piñeiro ${ }^{2} \&$ Yuzo Koketsu $^{1}$ \\ ${ }^{1}$ School of Agriculture, Meiji University, Kanagawa, Japan \\ ${ }^{2}$ PigCHAMP Pro Europa S.L., Segovia, Spain \\ Correspondence: Ryosuke Iida, School of Agriculture, Meiji University, Higashi-mita 1-1-1, Tama-ku, \\ Kawasaki, Kanagawa, 214-8571, Japan. Tel: 81-44-934-7826. E-mail: riid@shikon.meiji.ac.jp
}

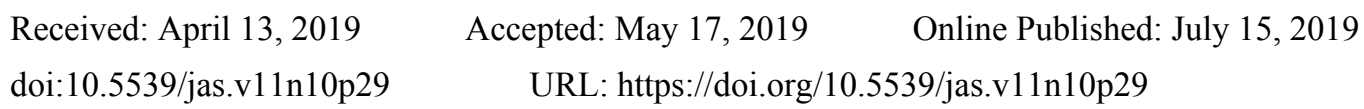

The research is financed by the Meiji University Graduate School International Joint Research Project Grant in Japan.

\begin{abstract}
Our objective was to characterize use of nurse sows in Spanish breeding herds. We analyzed 466111 parity records and lifetime records of 92716 sows farrowed between 2011 and 2017 in 69 herds having nurse records. Nurse sows were defined as sows that had weaned 2 or 3 litters in the same lactation period. Mixed-effects models were applied to the data to compare reproductive performance and lifetime productivity between nurse and non-nurse sows. Of all the sows, 6705 (7.2\%) sows served as nurse sows at least once in their lifetime, with $10.2 \%$ of the nurse sows having a second nurse event in a later parity. Mean values (SE) of lactation length and number of piglets weaned were $31.0(0.11)$ days and $21.9(0.04)$ piglets in nurse sows, respectively. Across parities 2-6, nurse sows had 1.9-3.0\% greater proportions of weaning-to-first-mating interval 7-20 days than non-nurse sows $(P<0.05)$. There was no difference between nurse sows and non-nurse sows in farrowing rate in any parity $(P \geq 0.13)$ and piglets born alive in parities $1-5(P \geq 0.15)$. Also, nurse sows had 3.7-7.4 more annualized lifetime piglets weaned than non-nurse sows $(P<0.01)$, because nurse sows had similar lifetime non-productive days with non-nurse sows $(P \geq 0.07)$, but produced 9.3-12.0 more lifetime piglets weaned than non-nurse sows $(P<0.01)$. Using nurse sows could be a good practice to cope with highly prolific sows.
\end{abstract}

Keywords: herd management, highly prolific sows, lifetime performance, porcine characteristics, swine

\section{Introduction}

The number of piglets born alive per litter (PBA) has been increasing in breeding herds during the last few decades (Andersson, Frössling, Engblom, Algers, \& Gunnarsson, 2016; Małopolska, Tuz, \& Lambert, 2018; Nielsen, Kristensen, \& Moustsen, 2018). This means that in some prolific sows the PBA can exceed the number of functional teats which is usually 14 (Klopfenstein, Farmer, \& Martineau, 2006; Amdi et al., 2017). One way to solve this problem is to use nurse sows, and this practice is intensively applied in Denmark and the Netherlands (Baxter et al., 2013). A nurse sow is a sow that weans its own litter and additional litters or excess piglets from other sows in a same lactation period.

A Danish study investigated the effects of nursing on subsequent reproductive performance, and found that the only negative effect was prolonged weaning-to-first-mating interval (Bruun et al., 2016). Prolonged WMI increases non-productive sow days which can result in a decreased sow lifetime productivity (Koketsu, Tani, \& Iida, 2017). It was also reported that sows with WMI 0-3 and 7 days or more had lower subsequent reproductive performance than those with WMI 4-6 days (Hoshino \& Koketsu, 2008). Furthermore, it is important to have a stable proportion of sows with WMI 0-6 days for breeding herd producers using a weekly group production system in order to achieve the target number of served sows per week (Kirkwood \& De Rensis, 2016). However, there have not been any reports about the effect of nursing on the proportions of WMI 0-3, 4-6, 7-20 and 21 days or more and their subsequent reproductive performance.

Spain is an important pig producing country, but scarce studies have assessed the performance of nurse sows from the first nurse events to their removal. Additionally, no single study has compared nurse sows and 
non-nurse sows in different parities for sow lifetime productivity, such as annualized lifetime piglets weaned. Thus, the objective in the present study was to characterize use of nurse sows in Spanish breeding herds.

\section{Method}

\subsection{Studied Herds}

A veterinary consultancy firm (PigCHAMP pro Europa S.L., Segovia, Spain) has accumulated a pig database by requesting all client producers to mail their data files on a regular basis. In July 2017, reproductive performance records and lifetime productivity records of sows in 155 Spanish client herds were extracted from the database between 2011 and 2017. Sixty-nine of the 155 herds that had nurse sow records used in this study. The gestation and lactation diets of sows in the studied herds were formulated by using cereals (barley, wheat and corn) and soybean meal. Replacement gilts in the herds were either purchased from breeding companies or home-produced through internal multiplication programs.

Spain is one of the major pig producing countries in Europe; there were 19630 breeding herds with 2568450 sows, and the average herd size was 131 sows in December 2013 (European Commission, 2017). In our study, the average herd size (SE) in 2011-2016 was 1013 (103.1) sows with a range of 80 to 3678 sows. Also, the average number of piglets weaned per sow per year was $25.4(0.25)$ piglets with a range of 19.3 to 30.0 piglets.

\subsection{Study Design and Exclusion Criteria}

The present study was designed as a retrospective cohort study, coordinating records of 94919 sows that were entered into the 69 herds between 2011 and 2013. The dataset included all the farrowing records (parity records) from 2011 to 2017 of the sows from their first-farrowing to removal.

At the time the data were collected, $1241(1.3 \%)$ sows were still active in the herds and so they were not used for analysis because they did not have lifetime records. Also, sows in the $99^{\text {th }}$ percentile for lifetime non-productive days were excluded (262 days or more; 908 sows). In addition, sows were excluded as incorrect records if they had a nurse sow record that showed only 1 litter weaned during a nursing event (54 sows). Thus, the lifetime records of the remaining 92716 sows were used for lifetime performance analyses (Dataset 1).

In 478562 parity records of these sows, records were excluded if they met any of the following criteria: total number of piglets born was 0 or 31 piglets or more (500 records; Bloemhof, Mathur, Knol, \& van der Waaij, 2013); WMI of 61 days or more (524 records; Marois, Brisbane, \& Laforest, 2000); lactation length of 0-9 days (6 167 records; Hoving, Soede, Graat, Feitsma, \& Kemp, 2011); and 0 piglets weaned (3 905 records). Parity records of nurse sow were also excluded if they had weaned 4 litters during a nursing event ( 7 records), because there were too few cases to analyze. In addition, parity records of non-nurse sow were excluded if they had lactation length of 42 days or more (1 348 records; Hoving, Soede, Graat, Feitsma, \& Kemp, 2011). Hence, 466 111 parity records of sows were used for analyses of reproductive performance (Dataset 2).

\subsection{Categories and Definitions}

Sows were categorized into two sow groups: nurse and non-nurse sows in each parity. Nurse sows were defined as sows that had weaned 2 or 3 litters in the same lactation period. Lifetime PBA and piglets weaned were the sums of the number of PBA and piglets weaned in a sow's lifetime, respectively. Annualized lifetime PBA and piglets weaned were defined as the lifetime PBA and piglets weaned, respectively, divided by the sum of reproductive herd life days, multiplied by 365 . Reproductive herd life days was defined as the time between the date of first service and the date of removal (Sasaki \& Koketsu, 2011). Lifetime non-productive days is the number of days when sows were neither gestating nor lactating in their reproductive herd life.

\subsection{Statistical Analysis}

All analyses were performed using SAS University Edition 2018 (SAS Inst. Inc., Cary, NC). All significance test levels were set at 0.05. Pairwise multiple comparisons were made using the Tukey-Kramer method.

Generalized linear mixed effect models were applied to the datasets by using MIXED and GLIMMIX procedure. Two statistical Models were created: Model 1 was applied to lifetime performance data (Dataset 1), and compared lifetime productivity between nurse and non-nurse sows; Model 2 was applied to reproductive performance data (Dataset 2), and compared sow reproductive performance between the two sow groups. Model 1 included the sow groups and entry year as fixed effects, whereas Model 2 included sow groups, quarterly farrowing month groups and entry year as fixed effects. A random herd effect was added to the both models to account for the clustering of sows within a herd. Model 1 was repeatedly constructed based on the sow groups in parity 1 to 6 . Model 2 was constructed by parity. 
A Fisher's exact test was used to access that distributions of the number of piglets weaned and lactation length differ between nurse sows that weaned two litters and three litters. Associations between lactation length in first litter and second litter of nurse sows were also examined.

\section{Results}

There were 6705 of the 92716 sows (7.2\%) that served as nurse sows at least once in their lifetime (Table 1) with 7442 nurse events in 466111 parity records (1.6\%). Mean total lactation length and total number of piglets weaned (SE) in nurse sows were $31.0(0.11)$ days and $21.9(0.04)$ piglets, respectively. Table 2 shows the parity of the first nurse events and what happened to the nurse sows after weaning. For example, of the 1223 sows that nursed in parity $1,13.1 \%$ had a second nurse event but $82.6 \%$ had no second nurse event in a later parity. Overall, $10.2 \%$ of the 6705 first nurse sows had a second nurse event in a later parity. Also, the proportion of nurse sows culled without any subsequent service increased from $3.9 \%$ in parity 1 to $66.6 \%$ in parity 6 or higher. Furthermore, 2988 of 6705 first nurse sows (44.6\%) were parity 5 or higher sows.

Of the 7442 nursing events, 98.5 and $1.5 \%$ weaned two and three litters, respectively. There were differences between the nurse sows that weaned two and three litters in the distributions of the total number of piglets weaned and total lactation length $(P<0.01$; Figure 1). For example, $70 \%$ of the nurse sows that weaned two litters produced 20-24 piglets weaned, whereas $70 \%$ of the nurse sows that weaned three litters produced 29-36 piglets weaned (Figure 1A). Also, $70 \%$ of the nurse sows that weaned two litters had total lactation length of 19-33 days, whereas $70 \%$ of the nurse sows that weaned three litters had lactation length of 22-42 days (Figure 1B). In addition, the frequency distributions (\%) of lactation length differed between the first and second litter in nurse sows that weaned two litters $(P<0.05$; Figure 2$)$. For example, $60 \%$ of the nurse sows had $18-24$ days of lactation length for the first litter, whereas for the second litter the lactation length of $60 \%$ of sows were only $3-9$ days.

Nurse sows had 3.8-8.6 days longer total lactation than non-nurse sows across parities $(P<0.01$; Table 3$)$. Also, nurse sows had 0.3-0.6 fewer PBA prior to the nurse events in parity 3 or higher, and had 0.2 more piglets weaned in the first lactation in parities 1,2 and 4 than non-nurse sows $(P<.05)$. With regard to reproductive performance after nurse event, there was no difference between nurse and non-nurse sows in farrowing rate in any parity $(P \geq 0.13)$ and in PBA in parities $1-5(P \geq 0.15)$. However, $2.5-19.9 \%$ more nurse sows than non-nurse sows were culled without subsequent service after parity $1(P<0.01)$. Nurse sows had 3.8-5.4 days longer farrowing-to-first-mating intervals than non-nurse sows across parities $(P<0.01)$.

The proportion of WMI 4-6 days was 3.4-10.0\% lesser in nurse sows than in non-nurse sows in parities 2 and 4 or higher, but the proportion of WMI 7-20 days was $1.9-3.1 \%$ greater in parity 2 or higher $(P<0.05$; Table 4$)$. In addition, nurse sows had 2.1-5.3\% greater proportions of WMI 0-3 days than non-nurse sows in parities 5 and 6 $(P<0.05)$.

Table 5 shows comparisons of lifetime productivity between nurse sows and non-nurse sows that were categorized in different parities. Sows that nursed in any parity had 9.3-12.0 more lifetime piglets weaned and 3.7-7.4 more annualized lifetime piglets weaned than non-nurse sows $(P<.001)$, but there was no difference between the groups for lifetime non-productive sow days $(P \geq 0.07)$. However, the parity at removal for sows that nursed in parities 5 and 6 was 0.2 lower than non-nurse sows $(P<0.01)$. Also, sows that nursed in parity 3 or higher had 1.6-3.5 fewer lifetime PBA, and sows that nursed in parity 2 or higher had 0.4-0.8 fewer annualized lifetime PBA than non-nurse sows $(P<0.05)$. 
Table 1. Lifetime and parity records of sows in 69 Spanish commercial breeding herds

\begin{tabular}{llll}
\hline Measurements & N & Mean (SE) & Medium (IQR) \\
\hline Lifetime records & & & \\
Sows that had nurse events, \% & 92716 & $7.2(0.09)$ & - \\
Parity at removal & 92716 & $5.2(0.01)$ & $6(3-7)$ \\
Lifetime piglets born alive & 92716 & $64.5(0.11)$ & $68(35-92)$ \\
Lifetime piglets weaned & 92716 & $56.4(0.10)$ & $61(32-80)$ \\
Lifetime non-productive days & 92716 & $66.9(0.16)$ & $52(31-90)$ \\
Annualized lifetime piglets born alive & 92716 & $29.0(0.02)$ & $30(25-33)$ \\
Annualized lifetime piglets weaned & 92716 & $25.4(0.02)$ & $26(23-29)$ \\
\hline Parity records & & & \\
Farrowed parity & 466111 & $3.7(0.01)$ & $3(2-5)$ \\
Piglets born alive prior to any nurse event & 466111 & $12.6(0.01)$ & $13(11-15)$ \\
Total number of piglets weaned & 466111 & $11.1(0.01)$ & $11(10-12)$ \\
Piglets weaned in the first litter of nurse sows in nursing parity* & 7330 & $11.0(0.02)$ & $11(10-12)$ \\
Piglets weaned in the second litter of nurse sows in nursing parity* & 7330 & $10.7(0.02)$ & $11(10-12)$ \\
Total lactation length, days & 466111 & $23.0(0.01)$ & $22(20-26)$ \\
First litter lactation length in nurse sows, days* & 7330 & $21.0(0.06)$ & $21(18-24)$ \\
Second litter lactation length in nurse sows, days* & 7330 & $9.8(0.08)$ & $7(5-14)$ \\
Farrowing-to-first-mating intervals, days & 407426 & $28.8(0.01)$ & $27(25-31)$ \\
Weaning-to-first-mating interval, days & 407426 & $5.8(0.01)$ & $5(4-5)$ \\
Proportion of weaning-to-first-mating interval 0-3 days, \% & 407426 & $7.7(0.04)$ & - \\
Proportion of weaning-to-first-mating interval 4-6 days, \% & 407426 & $81.2(0.06)$ & - \\
Proportion of weaning-to-first-mating interval 7-20 days, \% & 407426 & $7.8(0.04)$ & - \\
Proportion of weaning-to-first-mating interval 21 days or more, \% & 407426 & $3.3(0.03)$ & - \\
Farrowing rate, \% & 407426 & $88.4(0.05)$ & - \\
\hline
\end{tabular}

Note. SE: standard error; IQR: interquartile range.

* The remaining records $(7442-\mathrm{N})$ were nurse sows that weaned three litters, and there were no individual records of number of piglets weaned and lactation length for each litter.

Table 2. Subsequent service, second nurse events and removal information in 6705 sows that had a first nurse event in each parity

\begin{tabular}{|c|c|c|c|c|c|c|c|c|c|c|c|}
\hline \multirow{3}{*}{$\begin{array}{l}\text { Parity of first } \\
\text { nurse event }\end{array}$} & \multirow{3}{*}{$\mathrm{N}$} & \multicolumn{8}{|c|}{ Nurse sows with subsequent service } & \multirow{3}{*}{$\begin{array}{l}\text { Sows culled } \\
\text { without subsequent } \\
\text { service, } \%\end{array}$} & \multirow{3}{*}{$\begin{array}{l}\text { Sows that died } \\
\text { without subsequent } \\
\text { service, } \%\end{array}$} \\
\hline & & \multicolumn{7}{|c|}{$\begin{array}{c}\text { Sows with second nurse event (\%) } \\
\text { at each subsequent parity }\end{array}$} & \multirow{2}{*}{$\begin{array}{l}\text { Sows with } \\
\text { no second } \\
\text { nurse event, \% }\end{array}$} & & \\
\hline & & 2 & 3 & 4 & 5 & 6 & $\geq 7$ & Total & & & \\
\hline 1 & 1223 & 4.3 & 2.9 & 2.0 & 0.9 & 1.4 & 1.6 & 13.1 & 82.6 & 3.9 & 0.4 \\
\hline 2 & 925 & - & 4.1 & 3.7 & 2.8 & 2.1 & 2.3 & 14.9 & 78.6 & 5.7 & 0.8 \\
\hline 3 & 801 & - & - & 5.7 & 2.9 & 2.0 & 4.1 & 14.8 & 75.1 & 9.5 & 0.6 \\
\hline 4 & 768 & - & - & - & 4.4 & 3.8 & 3.6 & 11.9 & 75.5 & 11.9 & 0.7 \\
\hline 5 & 669 & - & - & - & - & 5.1 & 2.7 & 7.8 & 71.4 & 20.2 & 0.6 \\
\hline$\geq 6$ & 2319 & - & - & - & - & - & 5.3 & 5.3 & 27.1 & 66.6 & 1.0 \\
\hline Total & 6705 & 0.8 & 1.1 & 1.6 & 1.4 & 1.7 & 3.4 & 10.2 & 60.0 & 29.1 & 0.7 \\
\hline
\end{tabular}


Table 3. Comparisons of reproductive performance between nurse and non-nurse sows*

\begin{tabular}{|c|c|c|c|c|c|c|}
\hline Nurse groups & Parity 1 & Parity 2 & Parity 3 & Parity 4 & Parity 5 & Parity 6 \\
\hline Number of piglet. & prior to the $n$ & events & - & SE) ----- & -1-2--1 & - \\
\hline $\begin{array}{l}\text { Nurse sows } \\
\text { (No. of sows) }\end{array}$ & $\begin{array}{l}11.7(0.13) \\
(1220)\end{array}$ & $\begin{array}{l}12.2(0.13) \\
(976)\end{array}$ & $\begin{array}{l}12.6(0.13)^{b} \\
(875)\end{array}$ & $\begin{array}{l}12.8(0.13)^{\mathrm{b}} \\
(879)\end{array}$ & $\begin{array}{l}12.6(0.14)^{b} \\
(767)\end{array}$ & $\begin{array}{l}12.2(0.14)^{\mathrm{b}} \\
(949)\end{array}$ \\
\hline $\begin{array}{l}\text { Non-nurse sows } \\
\text { (No. of sows) }\end{array}$ & $\begin{array}{l}11.7(0.10) \\
(88944)\end{array}$ & $\begin{array}{l}12.4(0.08) \\
(79021)\end{array}$ & $\begin{array}{l}13.0(0.09)^{\mathrm{a}} \\
(71420)\end{array}$ & $\begin{array}{l}13.1(0.09)^{\mathrm{a}} \\
(63368)\end{array}$ & $\begin{array}{l}13.0(0.09)^{\mathrm{a}} \\
(55080)\end{array}$ & $\begin{array}{l}12.8(0.09)^{\mathrm{a}} \\
(45563)\end{array}$ \\
\hline \multicolumn{7}{|c|}{ Number of piglets weaned in the first lactation } \\
\hline $\begin{array}{l}\text { Nurse sows } \\
\text { (No. of sows) }\end{array}$ & $\begin{array}{l}11.1(0.10)^{\mathrm{a}} \\
(1220)\end{array}$ & $\begin{array}{l}11.2(0.10)^{\mathrm{a}} \\
(976)\end{array}$ & $\begin{array}{l}11.1(0.10) \\
(875)\end{array}$ & $\begin{array}{l}11.1(0.09)^{\mathrm{a}} \\
(879)\end{array}$ & $\begin{array}{l}10.9(0.10) \\
(767)\end{array}$ & $\begin{array}{l}10.6(0.10) \\
(949)\end{array}$ \\
\hline $\begin{array}{l}\text { Non-nurse sows } \\
\text { (No. of sows) }\end{array}$ & $\begin{array}{l}10.9(0.08)^{\mathrm{b}} \\
(88944)\end{array}$ & $\begin{array}{l}11.0(0.07)^{\mathrm{b}} \\
(79021)\end{array}$ & $\begin{array}{l}11.0(0.07) \\
(71420)\end{array}$ & $\begin{array}{l}10.9(0.07)^{\mathrm{b}} \\
(63368)\end{array}$ & $\begin{array}{l}10.8(0.07) \\
(55080)\end{array}$ & $\begin{array}{l}10.7(0.08) \\
(45563)\end{array}$ \\
\hline \multicolumn{7}{|c|}{ Total lactation length } \\
\hline $\begin{array}{l}\text { Nurse sows } \\
\text { (No. of sows) }\end{array}$ & $\begin{array}{l}28.1(0.33)^{\mathrm{a}} \\
(1220)\end{array}$ & $\begin{array}{l}28.2(0.31)^{\mathrm{a}} \\
(976)\end{array}$ & $\begin{array}{l}29.0(0.32)^{\mathrm{a}} \\
(875)\end{array}$ & $\begin{array}{l}28.8(0.32)^{\mathrm{a}} \\
(879)\end{array}$ & $\begin{array}{l}30.0(0.33)^{\mathrm{a}} \\
(767)\end{array}$ & $\begin{array}{l}32.5(0.35)^{\mathrm{a}} \\
(949)\end{array}$ \\
\hline $\begin{array}{l}\text { Non-nurse sows } \\
\text { (No. of sows) }\end{array}$ & $\begin{array}{l}24.3(0.32)^{b} \\
(88944)\end{array}$ & $\begin{array}{l}23.9(0.30)^{b} \\
(79021)\end{array}$ & $\begin{array}{l}23.9(0.30)^{b} \\
(71420)\end{array}$ & $\begin{array}{l}23.9(0.30)^{b} \\
(63368)\end{array}$ & $\begin{array}{l}23.9(0.31)^{b} \\
(55080)\end{array}$ & $\begin{array}{l}23.9(0.33)^{\mathrm{b}} \\
(45563)\end{array}$ \\
\hline \multicolumn{7}{|c|}{ Proportion of sows culled without subsequent service, \% } \\
\hline $\begin{array}{l}\text { Nurse sows } \\
\text { (No. of sows) }\end{array}$ & $\begin{array}{l}4.1(0.63) \\
(1220)\end{array}$ & $\begin{array}{l}6.0(0.83)^{\mathrm{a}} \\
(976)\end{array}$ & $\begin{array}{l}8.2(1.02)^{\mathrm{a}} \\
(875)\end{array}$ & $\begin{array}{l}10.4(1.21)^{\mathrm{a}} \\
(879)\end{array}$ & $\begin{array}{l}19.1(1.83)^{\mathrm{a}} \\
(767)\end{array}$ & $\begin{array}{l}34.2(0.03)^{\mathrm{a}} \\
(949)\end{array}$ \\
\hline $\begin{array}{l}\text { Non-nurse sows } \\
\text { (No. of sows) }\end{array}$ & $\begin{array}{l}4.3(0.27) \\
(88944)\end{array}$ & $\begin{array}{l}3.5(0.22)^{b} \\
(79021)\end{array}$ & $\begin{array}{l}4.2(0.37)^{b} \\
(71420)\end{array}$ & $\begin{array}{l}5.3(0.36)^{b} \\
(63368)\end{array}$ & $\begin{array}{l}7.8(0.53)^{b} \\
(55080)\end{array}$ & $\begin{array}{l}14.3(1.35)^{\mathrm{b}} \\
(45563)\end{array}$ \\
\hline \multicolumn{7}{|c|}{ Farrowing-to-first-mating intervals, days } \\
\hline $\begin{array}{l}\text { Nurse sows } \\
\text { (No. of sows) }\end{array}$ & $\begin{array}{l}35.5(0.45)^{\mathrm{a}} \\
(1 \quad 167)\end{array}$ & $\begin{array}{l}34.1(0.38)^{\mathrm{a}} \\
(911)\end{array}$ & $\begin{array}{l}34.3(0.39)^{\mathrm{a}} \\
(791)\end{array}$ & $\begin{array}{l}33.9(0.38)^{\mathrm{a}} \\
(775)\end{array}$ & $\begin{array}{l}33.9(0.40)^{\mathrm{a}} \\
(614)\end{array}$ & $\begin{array}{l}34.6(0.40)^{\mathrm{a}} \\
(528)\end{array}$ \\
\hline $\begin{array}{l}\text { Non-nurse sows } \\
\text { (No. of sows) }\end{array}$ & $\begin{array}{l}31.7(0.39)^{b} \\
(84170)\end{array}$ & $\begin{array}{l}29.8(0.33)^{\mathrm{b}} \\
(75981)\end{array}$ & $\begin{array}{l}29.7(0.33)^{b} \\
(67929)\end{array}$ & $\begin{array}{l}29.5(0.33)^{b} \\
(59456)\end{array}$ & $\begin{array}{l}29.4(0.34)^{b} \\
(50308)\end{array}$ & $\begin{array}{l}29.2(0.33)^{\mathrm{b}} \\
(37571)\end{array}$ \\
\hline \multicolumn{7}{|c|}{ Farrowing rate, \% } \\
\hline $\begin{array}{l}\text { Nurse sows } \\
\text { (No. of sows) }\end{array}$ & $\begin{array}{l}87.8(1.13) \\
(1167)\end{array}$ & $\begin{array}{l}89.7(1.13) \\
(911)\end{array}$ & $\begin{array}{l}88.6(1.24) \\
(791)\end{array}$ & $\begin{array}{l}87.3(1.26) \\
(775)\end{array}$ & $\begin{array}{l}90.1(1.23) \\
(614)\end{array}$ & $\begin{array}{l}86.2(1.63) \\
(528)\end{array}$ \\
\hline $\begin{array}{l}\text { Non-nurse sows } \\
\text { (No. of sows) }\end{array}$ & $\begin{array}{l}86.4(0.52) \\
(84170)\end{array}$ & $\begin{array}{l}88.9(0.44) \\
(75981)\end{array}$ & $\begin{array}{l}89.0(0.36) \\
(67929)\end{array}$ & $\begin{array}{l}88.7(0.37) \\
(59456)\end{array}$ & $\begin{array}{l}88.9(0.37) \\
(50308)\end{array}$ & $\begin{array}{l}87.9(0.42) \\
(37571)\end{array}$ \\
\hline \multicolumn{7}{|c|}{ Subsequent piglets born alive } \\
\hline $\begin{array}{l}\text { Nurse sows } \\
\text { (No. of sows) }\end{array}$ & $\begin{array}{l}12.4(0.12) \\
(1095)\end{array}$ & $\begin{array}{l}13.1(0.14) \\
(852)\end{array}$ & $\begin{array}{l}13.3(0.14) \\
(728)\end{array}$ & $\begin{array}{l}13.1(0.15) \\
(700)\end{array}$ & $\begin{array}{l}12.9(0.16) \\
(554)\end{array}$ & $\begin{array}{l}12.8(0.17)^{\mathrm{a}} \\
(476)\end{array}$ \\
\hline $\begin{array}{l}\text { Non-nurse sows } \\
\text { (No. of sows) }\end{array}$ & $\begin{array}{l}12.4(0.08) \\
(77952)\end{array}$ & $\begin{array}{l}13.0(0.09) \\
(70878)\end{array}$ & $\begin{array}{l}13.1(0.09) \\
(63142)\end{array}$ & $\begin{array}{l}13.0(0.08) \\
(54812)\end{array}$ & $\begin{array}{l}12.8(0.09) \\
(45718)\end{array}$ & $\begin{array}{l}12.5(0.10)^{\mathrm{b}} \\
(33025)\end{array}$ \\
\hline
\end{tabular}

Note. SE: standard error.

*Means and SE were estimated by using mixed models.

${ }^{\mathrm{ab}}$ Different superscripts within a column represent significant differences in means $(P<0.05)$. 
Table 4. Comparisons of four groups of proportions of weaning-to-first-mating interval between nurse and non-nurse sows*

\begin{tabular}{|c|c|c|c|c|c|c|}
\hline Nurse groups & Parity 1 & Parity 2 & Parity 3 & Parity 4 & Parity 5 & Parity 6 \\
\hline & \multicolumn{6}{|c|}{ 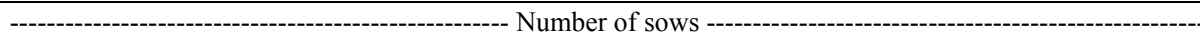 } \\
\hline Nurse sows & 1167 & 911 & 791 & 775 & 614 & 528 \\
\hline \multirow{2}{*}{ Non-nurse sows } & 84170 & 75981 & 67929 & 59456 & 50308 & 37571 \\
\hline & \multicolumn{6}{|c|}{ 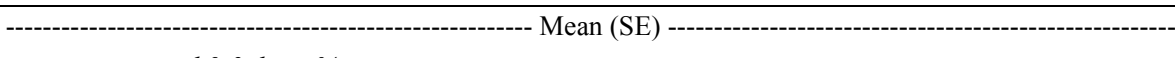 } \\
\hline \multicolumn{7}{|c|}{ Proportion of weaning-to-first-mating interval 0-3 days, \% } \\
\hline Nurse sows & $2.5(0.49)$ & $3.6(0.66)$ & $3.8(0.69)$ & $5.3(0.89)$ & $8.0(1.31)^{\mathrm{a}}$ & $11.6(1.84)^{\mathrm{a}}$ \\
\hline Non-nurse sows & $2.0(0.23)$ & $3.1(0.36)$ & $4.5(0.49)$ & $4.8(0.54)$ & $5.9(0.66)^{\mathrm{b}}$ & $6.3(0.72)^{b}$ \\
\hline \multicolumn{7}{|c|}{ Proportion of weaning-to-first-mating interval $4-6$ days, $\%$} \\
\hline Nurse sows & $76.8(1.87)$ & $80.5(1.70)^{\mathrm{b}}$ & $82.7(1.67)$ & $81.4(1.77)^{\mathrm{b}}$ & $77.8(2.22)^{\mathrm{b}}$ & $74.6(2.54)^{\mathrm{b}}$ \\
\hline Non-nurse sows & $78.5(1.19)$ & $85.1(0.83)^{\mathrm{a}}$ & $84.1(0.93)$ & $84.8(0.93)^{\mathrm{a}}$ & $84.1(1.11)^{\mathrm{a}}$ & $84.6(1.09)^{\mathrm{a}}$ \\
\hline \multicolumn{7}{|c|}{ Proportion of weaning-to-first-mating interval $7-20$ days, $\%$} \\
\hline Nurse sows & $11.3(1.37)$ & $10.3(1.27)^{\mathrm{a}}$ & $9.9(1.21)^{\mathrm{a}}$ & $8.2(1.13)^{\mathrm{a}}$ & $8.8(1.34)^{\mathrm{a}}$ & $7.5(1.30)^{\mathrm{a}}$ \\
\hline Non-nurse sows & $11.1(0.93)$ & $7.2(0.54)^{\mathrm{b}}$ & $7.1(0.48)^{\mathrm{b}}$ & $6.3(0.43)^{\mathrm{b}}$ & $5.9(0.47)^{\mathrm{b}}$ & $5.3(0.41)^{\mathrm{b}}$ \\
\hline \multicolumn{7}{|c|}{ Proportion of weaning-to-first-mating interval 21 days or more, $\%$} \\
\hline Nurse sows & $6.2(0.93)$ & $3.5(0.73)$ & $2.1(0.57)$ & $3.2(0.76)$ & $2.6(0.71)$ & $3.1(0.84)^{\mathrm{a}}$ \\
\hline Non-nurse sows & $5.5(0.45)$ & $2.6(0.22)$ & $2.4(0.20)$ & $2.2(0.19)$ & $2.0(0.19)$ & $1.8(0.17)^{\mathrm{b}}$ \\
\hline
\end{tabular}

Note. SE: standard error.

* Means and SE were estimated by using mixed models.

${ }^{\mathrm{ab}}$ Different superscripts within a column represent significant differences in means $(P<0.05)$.

Table 5. Comparisons of sow lifetime productivity between nurse sows and non-nurse sows*

\begin{tabular}{|c|c|c|c|c|c|c|}
\hline Nurse groups & Parity 1 & Parity 2 & Parity 3 & Parity 4 & Parity 5 & Parity 6 \\
\hline & \multicolumn{6}{|c|}{ - } \\
\hline Nurse sows & 1223 & 977 & 877 & 882 & 768 & 952 \\
\hline \multirow[t]{2}{*}{ Non-nurse sows } & 91493 & 80704 & 72764 & 64688 & 56262 & 46925 \\
\hline & \multicolumn{6}{|c|}{ 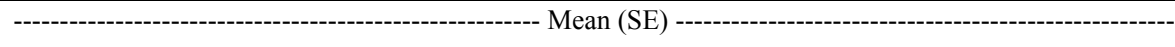 } \\
\hline \multicolumn{7}{|l|}{ Parity at removal } \\
\hline Nurse sows & $5.3(0.11)$ & $5.7(0.09)$ & $6.1(0.08)$ & $6.5(0.07)$ & $6.7(0.07)^{\mathrm{b}}$ & $7.1(0.06)^{\mathrm{b}}$ \\
\hline Non-nurse sows & $5.3(0.08)$ & $5.8(0.07)$ & $6.2(0.06)$ & $6.6(0.05)$ & $6.9(0.05)^{\mathrm{a}}$ & $7.3(0.05)^{\mathrm{a}}$ \\
\hline \multicolumn{7}{|c|}{ Lifetime piglets born alive } \\
\hline Nurse sows & $65.9(1.37)$ & $71.3(1.25)$ & $76.0(1.16)^{\mathrm{b}}$ & $80.4(1.06)^{\mathrm{b}}$ & $84.0(1.01)^{\mathrm{b}}$ & $89.0(0.92)^{\mathrm{b}}$ \\
\hline Non-nurse sows & $65.7(0.95)$ & $72.3(0.81)$ & $77.6(0.74)^{\mathrm{a}}$ & $82.6(0.72)^{\mathrm{a}}$ & $87.5(0.70)^{\mathrm{a}}$ & $92.5(0.71)^{\mathrm{a}}$ \\
\hline \multicolumn{7}{|c|}{ Lifetime piglets weaned } \\
\hline Nurse sows & $68.7(1.16)^{\mathrm{a}}$ & $73.4(1.05)^{\mathrm{a}}$ & $78.2(0.96)^{\mathrm{a}}$ & $82.4(0.87)^{\mathrm{a}}$ & $84.5(0.82)^{\mathrm{a}}$ & $88.9(0.74)^{\mathrm{a}}$ \\
\hline Non-nurse sows & $56.7(0.81)^{\mathrm{b}}$ & $62.5(0.70)^{\mathrm{b}}$ & $67.0(0.63)^{\mathrm{b}}$ & $71.1(0.61)^{\mathrm{b}}$ & $75.2(0.60)^{\mathrm{b}}$ & $79.3(0.59)^{\mathrm{b}}$ \\
\hline \multicolumn{7}{|c|}{ Lifetime non-productive days } \\
\hline Nurse sows & $71.1(2.29)$ & $74.7(2.41)$ & $75.1(2.49)$ & $73.7(2.46)$ & $71.6(2.52)$ & $73.1(2.41)$ \\
\hline Non-nurse sows & $71.7(1.80)$ & $73.4(1.84)$ & $73.7(1.88)$ & $73.4(1.89)$ & $72.3(1.93)$ & $70.3(1.96)$ \\
\hline \multicolumn{7}{|c|}{ Annualized lifetime piglets born alive } \\
\hline Nurse sows & $28.5(0.30)$ & $29.1(0.28)^{\mathrm{b}}$ & $29.3(0.28)^{\mathrm{b}}$ & $29.8(0.28)^{\mathrm{b}}$ & $30.3(0.29)^{\mathrm{b}}$ & $30.4(0.28)^{\mathrm{b}}$ \\
\hline Non-nurse sows & $28.7(0.23)$ & $29.5(0.22)^{\mathrm{a}}$ & $30.1(0.23)^{\mathrm{a}}$ & $30.5(0.23)^{\mathrm{a}}$ & $30.8(0.24)^{\mathrm{a}}$ & $31.2(0.25)^{\mathrm{a}}$ \\
\hline \multicolumn{7}{|c|}{ Annualized lifetime piglets weaned } \\
\hline Nurse sows & $32.2(0.27)^{\mathrm{a}}$ & $31.3(0.25)^{\mathrm{a}}$ & $31.0(0.25)^{\mathrm{a}}$ & $31.1(0.24)^{\mathrm{a}}$ & $30.7(0.24)^{\mathrm{a}}$ & $30.5(0.24)^{\mathrm{a}}$ \\
\hline Non-nurse sows & $24.8(0.21)^{\mathrm{b}}$ & $25.6(0.21)^{\mathrm{b}}$ & $26.1(0.22)^{\mathrm{b}}$ & $26.3(0.21)^{\mathrm{b}}$ & $26.5(0.22)^{\mathrm{b}}$ & $26.8(0.22)^{\mathrm{b}}$ \\
\hline
\end{tabular}

Note. SE: standard error.

* Means and SE were estimated by using mixed models.

${ }^{\mathrm{ab}}$ Different superscripts within a column represent significant differences in means $(P<0.05)$. 


\section{Discussion}

Our study clearly showed that nurse sows produced lifetime piglets weaned more efficiently than non-nurse sows, regardless of the nursing parity, but still had similar farrowing rates and PBA to non-nurse sows. These results suggest that the use of nurse sows may be a good practice in breeding herds to cope with the large numbers of piglets born per litter in highly prolific sows. This practice can also increase the number of lifetime piglets weaned in low prolific sows.

One concern regarding the use of nurse sows is the increase in WMI, reflected in our data by a lesser proportion of WMI 4-6 days and a greater proportion of WMI 7-20 days for nurse sows than for non-nurse sows in parity 2 or higher. These nurse sows would suffer from a loss of body weight or backfat thickness due to insufficient feed intake during the prolonged lactations (S. S. Anil, L. Anil, Deen, Baidoo, \& Walker, 2006). So, the prolonged WMI in pairty 2 or higher nurse sows could be suppressed by providing sufficient lactational feed. Meanwhile, the prolonged suckling intervals or changed suckling intensity when nurse sows receive a second or third litter could cause an occurrence of estrus during lactation (van Nieuwamerongen, Bolhuis, van der Peet-Schwering, \& Soede, 2014). Such prolonged suckling intervals or changed suckling intensity might be a cause of the increased occurrence of 0-3 days WMI in high parities in our study.

Our study showing the lack of any effect of nursing on farrowing rates and PBA, and negative effect on WMI in parity 2 or higher is consistent with a Danish study (Bruun et al., 2016). These counter-effects of nursing can be explained by the different reproductive mechanism between WMI and farrowing rates, or between WMI and PBA. The WMI is directly related to the estrus resumption which is highly related to gonadotropic secretion and follicle development in the hypothalamus-pituitary-ovary axis (Hoving, Soede, Graat, Feitsma, \& Kemp, 2011). In contrast, farrowing rates and PBA are not directly associated with this estrus resumption (Vinsky, Novak, Dixon, Dyck, \& Foxcroft, 2006; Bertoldo, Holyoake, Evans, \& Grupen, 2012). In addition, our study showed that nurse sows had longer farrowing-to-first-mating intervals than non-nurse sows. This could be due to prolonged lactation length and prolonged WMI in nurse sows, and cause the fewer annualized lifetime PBA in nurse sows than in non-nurse sows.

Sows that nursed in parity 1 had similar lifetime PBA and proportions of WMI 4-6 days to non-nurse sows in our study. This suggests that nurse sows are chosen as suitable sows to nurse a second litter based on their body reserve and feed intake, but not on PBA in their first litter. These sows could have enough body reserves and sufficient feed intake during the first lactation, and so still be able to resume normal estrus postweaning (Eissen, Apeldoorn, Kanis, Verstegen, \& de Greef, 2003).

Our study indicates that nurse sows that does not show postweaning estrus or had health problems during their lactation are culled without subsequent service. A previous study also reported a high risk of nurse sows having udder wounds and bursa on joint legs (Sørensen, Rousing, Kudahl, Hansted, \& Pedersen, 2016), which might be caused by the occurrence of teat fights by piglets (Schmitt, Baxter, Boyle, \& O'Driscoll, 2019), and by the prolonged stay in farrowing crate. Higher culling rate and lower parity at removal in nurse sows also can explain the discrepancy between the increased WMI in nurse sows but no difference between nurse and non-nurse sows in lifetime non-productive days.

The Spanish herds in our study had fewer nurse events than Danish herds where studies have reported parity records containing $20 \%$ or more nurse sow records (Hales, Moustsen, Nielsen, \& Hansen, 2015; Bruun et al., 2016). The herds in our study had fewer PBA than the Danish herds, and so there could be not such frequent use of nurse sows. Also, the second litter lactation length of 3-9 days for $60 \%$ of the nurse sows in our study is considerably shorter than Danish nurse sow strategy of having 3 weeks of lactation length (Bruun et al., 2016).

Our study suggests that selection of nurse sows depended on the number of piglets weaned in relatively low parity and on PBA in relatively late parity. In parities 1, 2 and 4, nurse sows weaned more piglets in their own litter than non-nurse sows. Meanwhile, in mid or late parities, producers chose nurse sows that had farrowed few PBA. These sows might be prospective sows to be culled due to low prolificacy or management reasons. The reason that only $10 \%$ of the nurse sows were repeatedly used as a nurse could be due to that approximately half of all nurse sows were parity 5 or higher sows. There are several other criteria for choosing nurse sows, such as sows having good body condition, normal or high feed intake and good nursing behavior (Bruun et al., 2016; Sørensen, Rousing, Kudahl, Hansted, \& Pedersen, 2016; Schmitt, Baxter, Boyle, \& O’Driscoll, 2019).

\section{Conclusion}

The nurse sows in our study appeared to be mainly used by producers to provide additional lactation for older piglets that failed to grow sufficiently or where the mother sow died or suffered agalactia (Alexopoulos, Lines, 
Hallett, \& Plush, 2018). However, this situation may change in the near future due to increasing PBA in more prolific sows. Nurse sows can produce more efficiently lifetime piglets weaned than non-nurse sows, while having no reduced farrowing rate, PBA and no prolonged lifetime non-productive days. The disadvantage of slightly greater proportion of WMI 7-20 days in parity 2 or higher nurse sows could be overcome by providing sufficient lactational feed for nurse sows and having the necessary numbers of replacement gilts available to meet the target number of served sows per week.

There were some limitations in this observational study, such as that our herd analyses did not survey genotype, nutrition, herd hygiene or semen quality. However, even with such limitations, this research provides worthful information for veterinarians and producers about the use of nurse sows to cope with highly prolific sows.
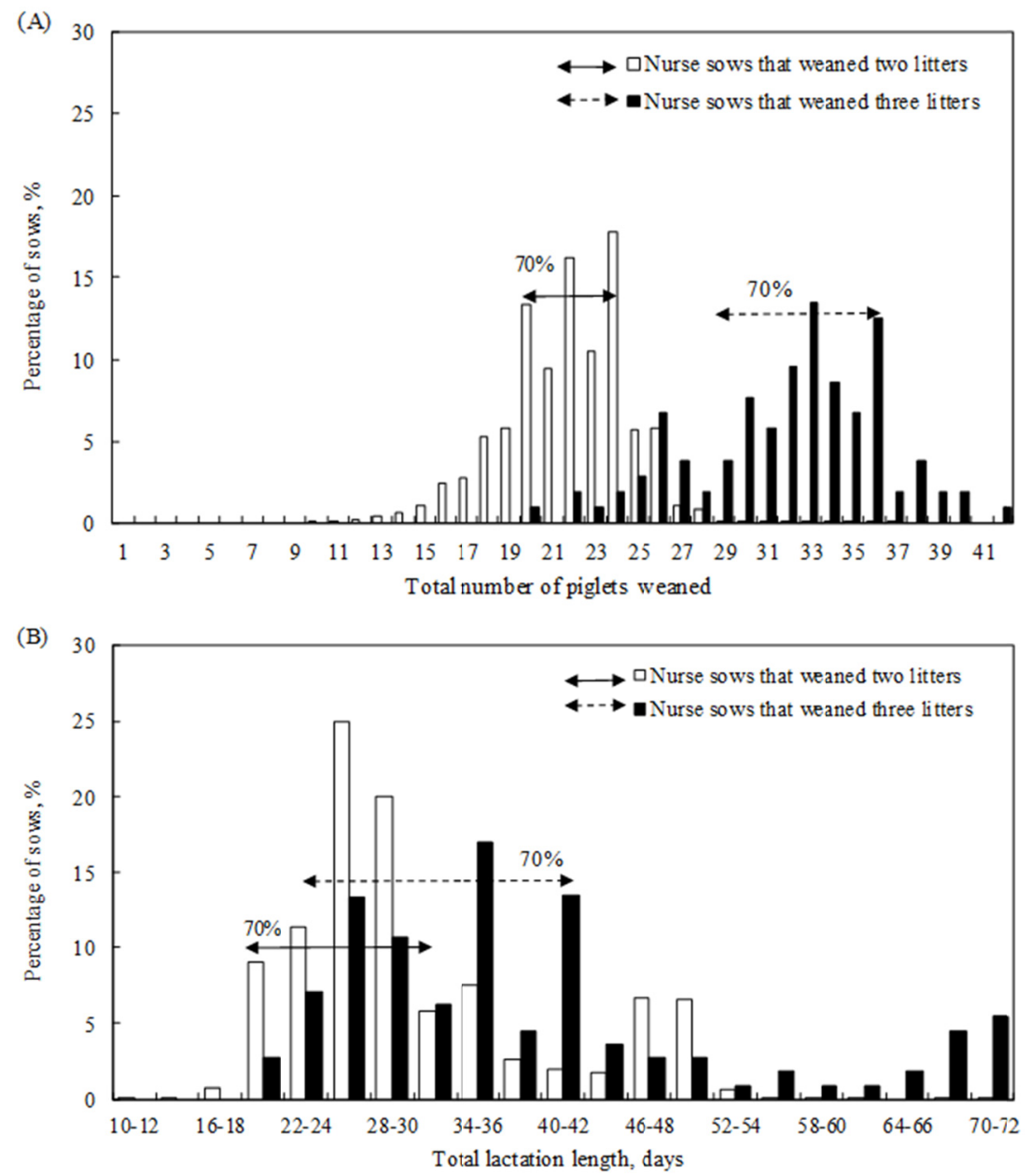

Figure 1. Relative frequency distributions (\%) of (A) the total number of piglets weaned and (B) total lactation length for 7330 parity records of nurse sows that weaned two litters, and 112 parity records of nurse sows that weaned three litters

Note. Frequencies within each group total $100 \%$. Relative frequency distributions differed between nurse sows that weaned two and three litters $(P<0.01$; Fisher's exact test). 


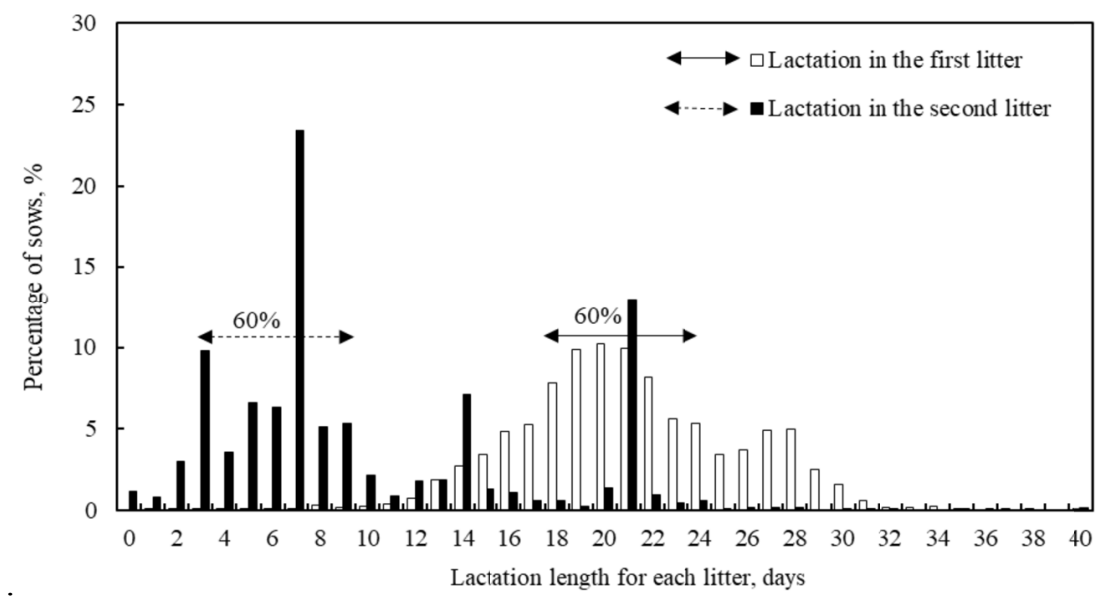

Figure 2. Relative frequency distributions (\%) of the lactation length in the first and second litter for 7330 parity records of nurse sows that weaned two litters

Note. Frequencies within each group total $100 \%$. Relative frequency distributions differed between lactation length in the first litter and second litter $(P<0.05$; Fisher's exact test $)$.

\section{Acknowledgements}

This work was supported by the Meiji University Graduate School International Joint Research Project Grant in Japan. The authors gratefully thank the swine producers for their cooperation in providing their valuable data for use in this study. We also thank Dr. I. McTaggart for his critical review of this manuscript.

\section{References}

Alexopoulos, J. G., Lines, D. S., Hallett, S., \& Plush, K. J. (2018). A Review of Success Factors for Piglet Fostering in Lactation. Animals, 8(3). https://doi.org/10.3390/ani8030038

Amdi, C., Moustsen, V. A., Oxholm, L. C., Baxter, E. M., Sørensen, G., Eriksson, K. B., ... Hansen, C. F. (2017) Comparable cortisol, heart rate and milk let-down in nurse sows and non-nurse sows. Livest. Sci., 198, 174-181. https://doi.org/10.1016/j.livsci.2017.02.024

Andersson, E., Frössling, J., Engblom, L., Algers, B., \& Gunnarsson, S. (2016). Impact of litter size on sow stayability in Swedish commercial piglet producing herds. Acta Vet. Scand., 58(1), 31-31. https://doi.org/ 10.1186/s13028-016-0213-8

Anil, S. S., Anil, L., Deen, J., Baidoo, S. K., \& Walker, R. D. (2006). Association of inadequate feed intake during lactation with removal of sows from the breeding herd. J. Swine Health Prod., 14(6), 296-301.

Baxter, E., Rutherford, K., d'Eath, R., Arnott, G., Turner, S., Sandøe, P., ... Lawrence, A. (2013). The welfare implications of large litter size in the domestic pig II: Management factors. Anim. Welfare, 22(2), 219-238. https://doi.org/10.7120/09627286.22.2.219

Bertoldo, M. J., Holyoake, P. K., Evans, G., \& Grupen, C. G. (2012). Seasonal variation in the ovarian function of sows. Reprod. Fert. Develop., 24(6), 822-834. https://doi.org/10.1071/RD11249

Bloemhof, S., Mathur, P. K., Knol, E. F., \& van der Waaij, E. H. (2013). Effect of daily environmental temperature on farrowing rate and total born in dam line sows. J. Anim. Sci., 91(6), 2667-2679. https://doi.org/10.2527/jas.2012-5902

Bruun, T. S., Amdi, C., Vinther, J., Schop, M., Strathe, A. B., \& Hansen, C. F. (2016). Reproductive performance of "nurse sows" in Danish piggeries. Theriogenology, 86(4), 981-987. https://doi.org/ 10.1016/j.theriogenology.2016.03.023

Eissen, J. J., Apeldoorn, E. J., Kanis, E., Verstegen, M. W., \& de Greef, K. H. (2003). The importance of a high feed intake during lactation of primiparous sows nursing large litters. J. Anim. Sci., 81(3), 594-603. https://doi.org/10.2527/2003.813594x

European Commission. (2017). Pig: Number of farms and heads by agricultural size of farm (UAA) and size of pig herd. Retrieved April 1, 2019, from http://ec.europa.eu/eurostat/web/products-datasets/-/ef_lspigaa 
Hales, J., Moustsen, V. A., Nielsen, M. B., \& Hansen, C. F. (2015). Temporary confinement of loose-housed hyperprolific sows reduces piglet mortality. J. Anim. Sci., 93(8), 4079-4088. https://doi.org/10.2527/ jas.2015-8973

Hoshino, Y., \& Koketsu, Y. (2008). A repeatability assessment of sows mated 4-6 days after weaning in breeding herds. Anim. Reprod. Sci., 108(1), 22-28. https://doi.org/10.1016/j.anireprosci.2007.06.029

Hoving, L. L., Soede, N. M., Graat, E. A. M., Feitsma, H., \& Kemp, B. (2011). Reproductive performance of second parity sows: Relations with subsequent reproduction. Livest. Sci., 140(1-3), 124-130. http://doi.org/10.1016/j.livsci.2011.02.019

Kirkwood, R. N., \& De Rensis, F. (2016). Control of estrus in gilts and primiparous sows. Thai J. Vet. Med., 46(1), 1-7. Retrieved from https://www.tci-thaijo.org/index.php/tjvm/article/view/49777/41195

Klopfenstein, C., Farmer, C., \& Martineau, G. (2006). Diseases of the mammary glands. In B. E. Straw, J. Zimmerman, S. D’Allaire, \& D. J. Taylor (Eds.), Diseases of Swine (Vol. 9, pp. 57-86). Ames, IA, USA: Iowa State Univ. Press.

Koketsu, Y., Tani, S., \& Iida, R. (2017). Factors for improving reproductive performance of sows and herd productivity in commercial breeding herds. Porcine Health Manag., 3(1), 1. https://doi.org/10.1186/ s40813-016-0049-7

Małopolska, M. M., Tuz, R., \& Lambert, B. D. (2018). The replacement gilt: Current strategies for improvement of the breeding herd. J. Swine Health Prod., 26(4), 208-214. Retrieved from https://www.aasv.org/ shap/issues/v26n4/v26n4p208.pdf

Marois, D., Brisbane, J. R., \& Laforest, J. P. (2000). Accounting for lactation length and weaning-to-conception interval in genetic evaluations for litter size in swine. J. Anim. Sci., 78(7), 1796-1810. https://doi.org/ $10.2527 / 2000.7871796 x$

Nielsen, S. E., Kristensen, A. R., \& Moustsen, V. A. (2018). Litter size of Danish crossbred sows increased without changes in sow body dimensions over a thirteen year period. Livest. Sci., 209, 73-76. https://doi.org/10.1016/j.livsci.2018.01.015

Sasaki, Y., \& Koketsu, Y. (2011). Reproductive profile and lifetime efficiency of female pigs by culling reason in high-performing commercial breeding herds. J. Swine Health Prod., 19(5), 284-291. Retrieved from https://www.aasv.org/shap/issues/v19n5/v19n5p284.pdf

Schmitt, O., Baxter, E. M., Boyle, L. A., \& O’Driscoll, K. (2019). Nurse sow strategies in the domestic pig: II. Consequences for piglet growth, suckling behaviour and sow nursing behaviour. Animal, 13(3), 590-599. https://doi.org/10.1017/s1751731118001702

Sørensen, J. T., Rousing, T., Kudahl, A. B., Hansted, H. J., \& Pedersen, L. J. (2016). Do nurse sows and foster litters have impaired animal welfare? Results from a cross-sectional study in sow herds. Animal, 10(4), 681-686. https://doi.org/10.1017/s1751731115002104

van Nieuwamerongen, S. E., Bolhuis, J. E., van der Peet-Schwering, C. M., \& Soede, N. M. (2014). A review of sow and piglet behaviour and performance in group housing systems for lactating sows. Animal, 8(3), 448-460. https://doi.org/10.1017/s1751731113002280

Vinsky, M., Novak, S., Dixon, W., Dyck, M., \& Foxcroft, G. (2006). Nutritional restriction in lactating primiparous sows selectively affects female embryo survival and overall litter development. Reprod. Fertil. Dev., 18(3), 347-355. https://doi.org/10.1071/RD05142

\section{Copyrights}

Copyright for this article is retained by the author(s), with first publication rights granted to the journal.

This is an open-access article distributed under the terms and conditions of the Creative Commons Attribution license (http://creativecommons.org/licenses/by/4.0/). 